\title{
Study on the Relationship Between Air Pollution and Economic Development
}

\author{
Yang Zhanhong, Luo Hong, Wang Xiao \\ Department of Environment \& Economy, Chinese Research Academy of Environmental Sciences, Beijing, China
}

Email address:

yangzh@craes.org.cn (Yang Zhanhong), luohong@craes.org.cn (Luo Hong), wangxiao@ craes.org.cn (Wang Xiao)

\section{To cite this article:}

Yang Zhanhong, Luo Hong, Wang Xiao. Influence of Titanium Carbide on the Three - Body Abrasive Wear Behaviour of Glass-Fabric Reinforced Epoxy Composites. Science Discovery. Vol. 6, No. 1, 2018, pp. 12-18. doi: 10.11648/j.sd.20180601.13

Received: January 19, 2018; Accepted: February 1, 2018; Published: March 9, 2018

\begin{abstract}
In recent years, the government has adopted a series of measures to control air pollution, and the atmospheric environment quality has been improved. However, the environmental air quality in China is still not ideal. In this paper, constructs a regression model based on the EKC hypothesis, and builds a Tapio decoupling model based on the decoupling theory. The relationships between atmospheric pollutants and GDP are analyzed by using thses two methods. The atmospheric pollutants include $\mathrm{SO}_{2}, \mathrm{NOx}$ and $\mathrm{CO}_{2}$. In the regression model, the quantitative relationship between atmospheric pollutants and GDP is established, and the characteristics and differences between the pollutants and GDP are analyzed. Also, the decoupling relationship between atmospheric pollutants and economic growth is analyzed by using decoupling model. Then, the mutual verification of these different methods are launched. On this basis, the new situation of air pollution in China under the background of ecological civilization construction is analyzed. At last, policy suggestions are put forward.
\end{abstract}

Keywords: EKC, Economic Growth, Air Pollution, Decoupling Analysis, $\mathrm{CO}_{2}$

\section{大气污染与经济发展的关系研究}

\section{杨占红，罗宏，王晓}

环境与经济研究室, 中国环境科学研究院, 北京, 中国

\section{邮箱}

yangzh@craes.org.cn（杨占红）, luohong@craes.org.cn（罗宏）, wangxiao@ craes.org.cn（王晓）

摘要: 近年来, 国家对大气污染采取了一系列治理措施, 大气环境得到一定改善, 但目前中国的环境空气质量状况仍 不理想。本文通过构建基于EKC假说的回归模型和脱钩理论的 Tapio脱钩模型, 以两种方法分别对 $\mathrm{SO}_{2} 、 \mathrm{NOx} 、 \mathrm{CO}_{2}$ 与 经济增长的关系进行相关性分析。回归模型中, 建立 $\mathrm{SO}_{2} 、 \mathrm{NOx} 、 \mathrm{CO}_{2}$ 与 GDP之间的量化关系, 分析各污染物与GDP之 间关系的特点与异同; 利用脱钩模型, 分析 $\mathrm{SO}_{2} 、 \mathrm{NOx} 、 \mathrm{CO}_{2}$ 与经济增长的脱钩关系, 并与回归模型相互印证。在此基 础上，分析生态文明建设的背景下中国大气污染面临的新形势，提出应对建议。

关键词: $\mathrm{EKC}$, 经济增长, 大气污染, 脱钩分析, $\mathrm{CO}_{2}$ 


\section{1. 引言}

在当前经济发展模式下, 经济的发展一般会伴随着资 源消耗的增加和污染物排放的增加, 大气污染也会对经济 的发展产生影响。大气环境污染已成为经济发展、能源消 费的重要约束条件。如何在发展经济的同时保护大气环境 质量, 是当前需要解决的重要问题。

\section{2. 文献综述}

大气污染物排放与经济发展的关系, 与中国经济发展 方式有关, 也与大气污染防治的各项措施有关。国内外对 大气污染与经济的关系开展了多项研究, 如Bruvoll and Medin(2000)分析了1990-1999年挪威天气污染与经济增长 的关系变化, 认为经济增长导致了大气污染排放量增加了 $60 \%$ [1], Bernard等 (2011) 从二氧化碳和二氧化硫两种 污染物出发证实了 OECD国家存在倒U型关系 $[2,3]$, 还有 学者通过 $E K C$ 假说, 认为环境污染的下降是由技术或人力 资本对环境资源要素的替代所引起的[4], Dogan选取欧盟 对 $\mathrm{CO}_{2}$ 与经济的关系验证了 $\mathrm{EKC}$ 假说, Menendez等对环境 成本和可再生资源进行了 $\mathrm{EKC}$ 假说的验证, Hauff等对印 度的饮用水及地下水等进行了 $\mathrm{EKC}$ 验证 [5 7]。De Bruyn(2000)将脱钩分成强脱钩和弱脱钩 [8], Vehmas 等 (2003) 将脱钩划分为强脱钩、弱脱钩、强复钩、弱复钩和 扩张性复钩, 并考虑到经济衰退提出了衰退性脱钩的概念 [9], Tapio进一步地将脱钩以弹性系数的形式呈现出来, 将经济增长与污染物排放以率的变化, 将脱钩系数进行了 划分 [10], Gray等对交通运输排放的 $\mathrm{CO}_{2}$ 与经济增长进行 了脱钩分析 [11]。国外的研究表明, 在前期研究中, 经济 的增长导致了污染的增加, 但后期的部分研究表明, 经济 发展与污染排放倒U型曲线和脱钩关系的存在, 且研究对 象逐渐向新的污染物或者其他资源扩展。

国内学者运用两种理论从不同角度分析研究了环境 污染与经济增长的关系，包括从不同区域、不同污染物、 不同时间段等，如王敏和黄漟（2015）对2003-2010年中 国112个城市的研究发现, 城市大气污染与经济增长之间 存在倒U型关系[12], 范丹 (2014) 通过建立EKC曲线扩 展模型对中国 $\mathrm{CO}_{2}$ 与经济的关系做了研究 [13], 罗波等 （2017）、尚杰等（2017）、王光升等（2014）分别对资 源富集区、农业领域、沿海地区的经济增长与污染的关系 做了EKC验证 [14 16]。脱钩理论主要解释经济增长与污染 排放是否同步变化的关联性, 国内学者也分别从经济增长 与污染排放、与碳排放、与工业污染物排放的脱钩关系, 以及城市等区域的经济发展与环境污染的脱钩关系进行 了研究[17 21]。

国内外的研究基本都以传统的污染物或者碳与经济 的关系进行研究, 未对两者的进行统一比较分析。中国污 染控制 $\mathrm{SO}_{2}$ 和 $\mathrm{NO}_{\mathrm{x}}$ 前后被列为总量控制指标之一, 并在实 施总量控制后, 实现年排放量的逐年下降, 但 $\mathrm{CO}_{2}$ 的排放 控制, 仍以排放强度的下降程度进行考核。本文以 $\mathrm{SO}_{2}$ 、 $\mathrm{NO}_{\mathrm{x}} 、 \mathrm{CO}_{2}$ 为大气污染替代变量, 通过分析 $\mathrm{SO}_{2} 、 \mathrm{NO}_{\mathrm{x}} 、 \mathrm{CO}_{2}$ 三者之间与经济增长关系的量化关系及相互之间的异同,
可在一定程度反应各污染物的治理效果, 并为后续的污染 因子治理提供借鉴经验, 如 $\mathrm{NOx}$ 控制对 $\mathrm{SO}_{2}$ 治理经验的借 鉴, 以及 $\mathrm{CO}_{2}$ 控制对 $\mathrm{NOx}$ 和 $\mathrm{SO}_{2}$ 治理经验的借鉴, 并通过 分析各污染物与经济增长的关系, 结合目前的大气污染防 治新形势, 提出中国新时期大气污染防治的应对建议。

\section{3. 数据与方法}

\section{1. 研究数据}

本文使用的数据中, 经济数据以不变价GDP代表经济 增长, 主要来源于历年的《中国统计年鉴》; 大气污染本 身是一个难以量化的指标, 本文以 $\mathrm{SO}_{2} 、 \mathrm{NOx}$ 和 $\mathrm{CO}_{2}$ 作为 大气污染的替代变量, $\mathrm{SO}_{2} 、 \mathrm{NOx}$ 主要来自历年的《环境 统计年报》, $\mathrm{CO}_{2}$ 来自《BP Statistical Review of World Energy June 2016》。样本的时间跨度为2000年-2015年。 样本区间内, 中国 $\mathrm{SO}_{2} 、 \mathrm{NOx} 、 \mathrm{CO}_{2}$ 排放量与 GDP增长情 况见表1和图1。

表1 中国GDP及大气污染物排放数据。

\begin{tabular}{lllll}
\hline 年份 & GDP (亿元) & $\begin{array}{c}\mathrm{SO}_{2} \text { 排放量 } \\
\text { (万吨) }\end{array}$ & $\begin{array}{c}\text { NOx排放量 } \\
\text { (万吨) }\end{array}$ & $\begin{array}{c}\mathrm{CO}_{2} \text { 排放量 } \\
\text { (千万吨) }\end{array}$ \\
\hline 2000 & 150269.2 & 1995.1 & - & 335.2671 \\
2001 & 162434.8 & 1947.8 & - & 351.4958 \\
2002 & 178022 & 1926.6 & - & 383.4211 \\
2003 & 196670.6 & 2158.5 & - & 452.2606 \\
2004 & 217340.1 & 2254.9 & - & 532.3306 \\
2005 & 241031.1 & 2549.4 & - & 608.3598 \\
2006 & 273105.8 & 2588.8 & 1523.8 & 666.1573 \\
2007 & 313264.3 & 2468.1 & 1643.4 & 722.3889 \\
2008 & 344838.9 & 2321.2 & 1624.3 & 736.2312 \\
2009 & 374292.5 & 2214.4 & 1692.7 & 769.2532 \\
2010 & 413030.3 & 2185.1 & 1852.4 & 811.8674 \\
2011 & 452429.9 & 2217.9 & 2404.274 & 880.6716 \\
2012 & 487976.2 & 2117.6 & 2337.762 & 897.938 \\
2013 & 525835.4 & 2043.9 & 2227.359 & 921.8752 \\
2014 & 564194.4 & 1974.0 & 2078.002 & 922.4102 \\
2015 & 603212.1 & 1859.1 & 1851.024 & 916.4453 \\
2016 & 643627.3 & 1102.9 & 1394.311 & 912.3049 \\
\hline
\end{tabular}

注: GDP以2010年不变价计, NOx自2006年开始全国范围统计。

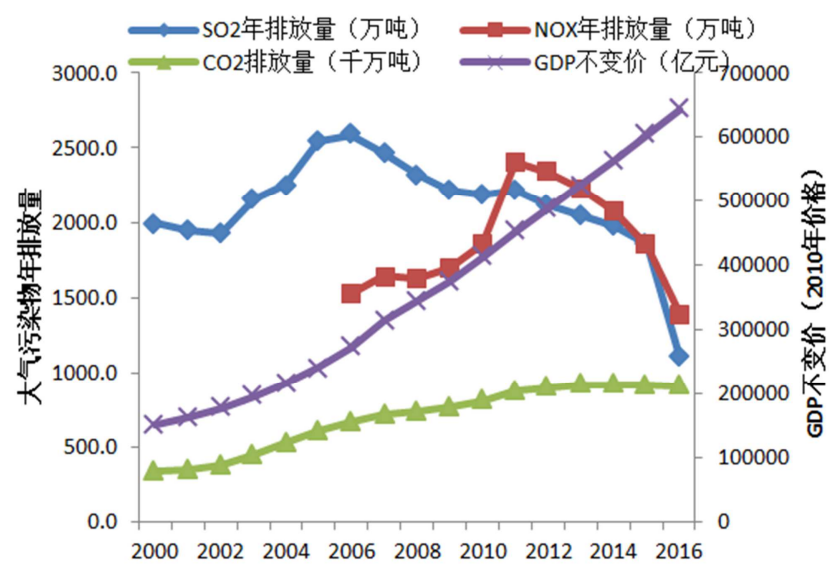

图1 中国大气污染物排放与经济发展趋势图。 


\section{2. 模型构建}

本文试图在已有的研究基础上, 通过构建回归模型和 Tapio脱钩模型, 以两种方法分别对 $\mathrm{SO}_{2} 、 \mathrm{NOx} 、 \mathrm{CO}_{2}$ 与经 济增长的关系进行分析验证, 建立 $\mathrm{SO}_{2} 、 \mathrm{NOx} 、 \mathrm{CO}_{2}$ 与 GDP 之间的量化关系, 分析各污染物与GDP之间关系的特点与 异同，并进一步对比两种方法结论的吻合性。

\section{2. 1. EKC模型构建}

环境库兹涅茨曲线理论 (Environmental Kuznets Curve, $\mathrm{EKC}$ ）, 又称“倒U曲线”, 最开始是为研究经济发展而提 出的[22], 之后, Grossman 和Kruger (1991) 将此理论应 用到了环境污染与经济发展的关系中, 指出经济增长和环 境污染会呈现一种倒U型曲线关系, 即在追求经济过快增 长中, 忽略环境的保护, 引起环境污染加重, 但当经济增 长到一定水平后, 污染程度达到峰值, 之后又会随着经济 的进一步增加, 人们开始加强环境的保护, 污染程度逐渐 降低, 进而呈现倒U形[23]。

大气污染的驱动因素有很多, 包括GDP产值、能源消 费、科技进步、产业结构、污染防治措施等等, 而各项因 素也会影响GDP产值, 相互关联性导致污染物的排放与 GDP可能不会呈现简单的直线或者倒U形曲线关系。本文 尝试建立大气污染排放与GDP的高阶曲线关系, 以经济发 展指标为横坐标, 以环境指标为纵坐标, 采用简化的一元 高次幂函数方程, 公式为:

$$
y=\beta_{0}+\beta_{1} x+\beta_{2} x^{2}+\cdots+\beta_{n} x^{n}+\xi
$$

其中, $\mathrm{y}$ 表示 $\mathrm{SO}_{2} 、 \mathrm{NOx}$ 和 $\mathrm{CO}_{2}$ 等污染物排放量, $\mathrm{x}$ 表 示地区生产总值, $\beta_{\mathrm{n}}(\mathrm{n}=0,1,2 \ldots \ldots)$ 为模型的参数, 它 们取值的不同, 反映经济增长与大气污染排放之间的关系。 自变量GDP以不变价（2010年价格）计算，以 $\mathrm{SO}_{2} 、 \mathrm{NOx}$ 和 $\mathrm{CO}_{2}$ 排放总量为因变量, 研究经济增长与污染物排放之 间的关系，通过回归模拟，建立函数关系。

\subsubsection{Tapio脱钩模型构建}

根据EKC假说, 经济的增长一般带来环境的污染和资 源的消耗, 但当采取一些有效措施后, 可能会以较低的环 境代价换来经济的增长, 这个过程被称为脱钩, 即 $\mathrm{EKC}$ 曲 线跨越拐点的后半段。脱钩理论是经济合作与发展组织

(OECD) 提出的, 用于分析经济发展与资源消耗或环境 污染之间的关系 [24]。本文利用Tapio脱钩模型 [10], 对 2000 年以来中国大气污染排放和经济发展统计数据之间的脱 钩关系进行测度。在Tapio脱钩模型中, 将弹性指数 $\mathrm{e}$ 作为 主要脱钩指标, 计算公式为:

$$
\begin{aligned}
& \mathrm{e}\left(\mathrm{SO}_{2}, \mathrm{GDP}\right)=\left(\frac{\Delta \mathrm{SO}_{2}}{\mathrm{SO}_{2}}\right) /\left(\frac{\Delta \mathrm{GDP}}{\mathrm{GDP}}\right) \\
& \mathrm{e}\left(\mathrm{NO}_{\mathrm{x}}, \mathrm{GDP}\right)=\left(\frac{\Delta \mathrm{NO}_{\mathrm{x}}}{\mathrm{NO}_{\mathrm{x}}}\right) /\left(\frac{\Delta \mathrm{GDP}}{\mathrm{GDP}}\right) \\
& \mathrm{e}\left(\mathrm{CO}_{2}, \mathrm{GDP}\right)=\left(\frac{\Delta \mathrm{CO}_{2}}{\mathrm{CO}_{2}}\right) /\left(\frac{\Delta \mathrm{GDP}}{\mathrm{GDP}}\right)
\end{aligned}
$$

根据Tapio对弹性指数的划分, 本文将弹性指数值根 据脱钩或未脱钩划分为 6 种类型, 如图 1 所示。其中强脱钩
是实现经济低碳化发展的最理想状态; 相应地强负脱钩为 最不利状态。

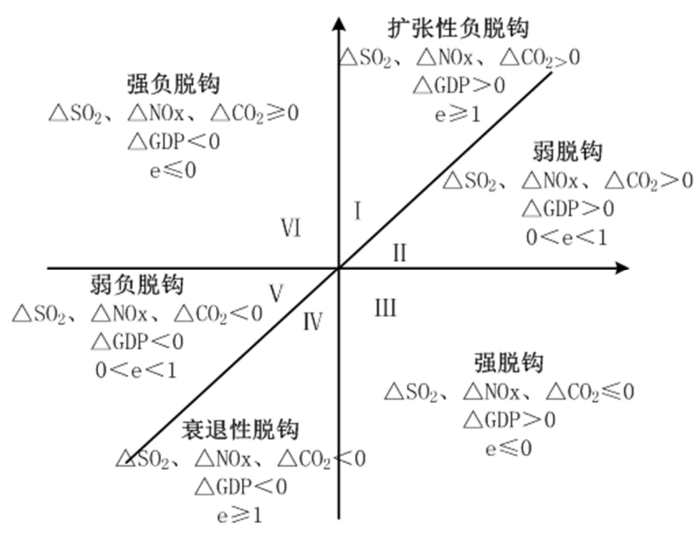

图2 大气污染物排放与经济增长脱钩分析模型。

\section{4. 结果讨论}

\subsection{EKC分析结果}

(1) 简化的EKC曲线分析

经分析验证，中国 $\mathrm{SO}_{2}$ 和 $\mathrm{NOx}$ 排放与 GDP呈现倒U关 系, 简化的 $\mathrm{EKC}$ 曲线关系如图所示, 对应的关系方程式及 关系曲线见图3 和图 4, 拟合相关系数一般, 分别为 0.7928 和 0.7031 。而 $\mathrm{CO}_{2}$ 与 $\mathrm{GDP}$ 呈正向直线关系, 对应的关系图 及关系方程式见图 5 , 拟合系数为 0.8802 。

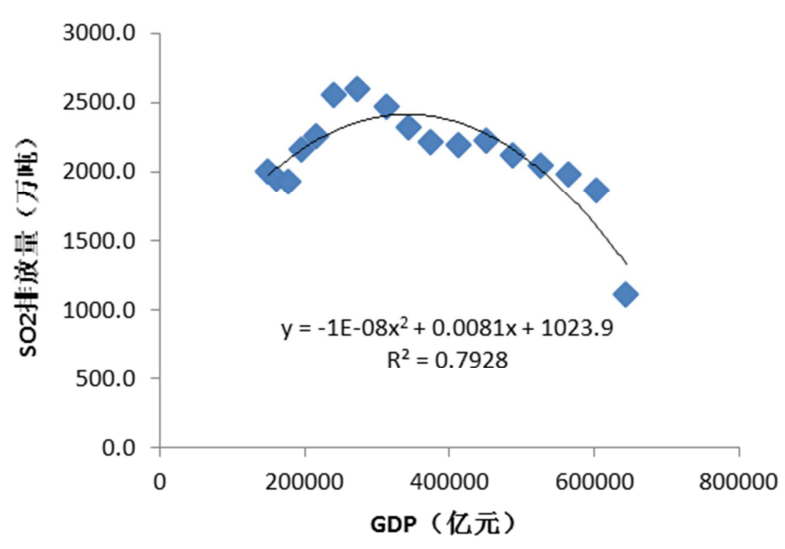

图3 $\mathrm{SO}_{2}$ 排放与 GDP的简化关系曲线。

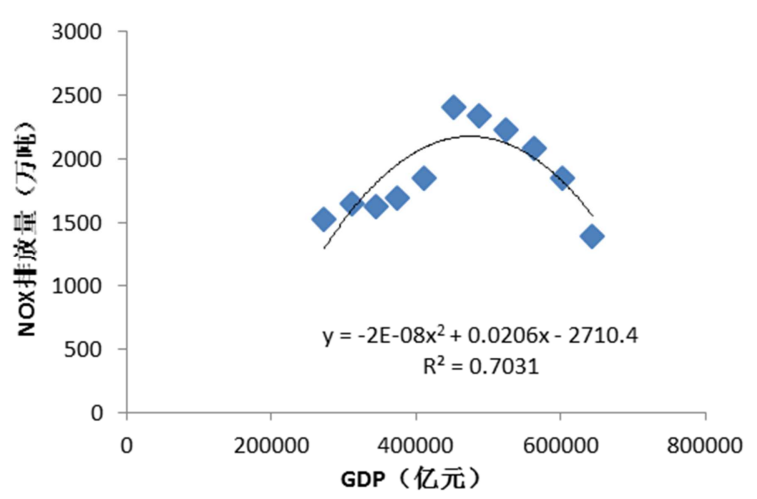

图4 NOx排放与GDP的简化关系曲线。 


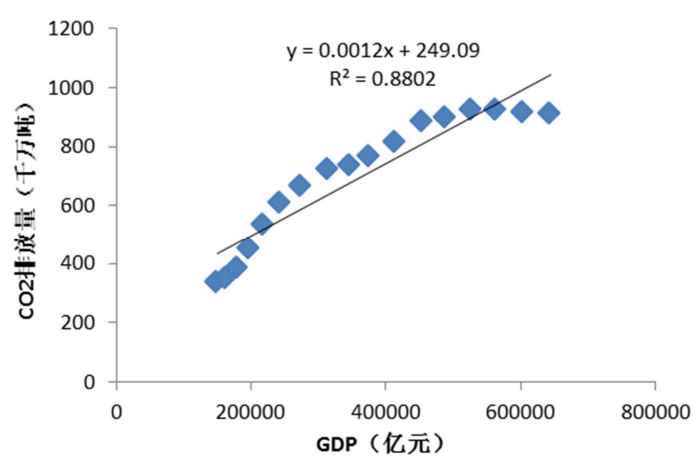

图5 $\mathrm{CO}_{2}$ 排放与 $\mathrm{GDP}$ 的简化关系曲线。
（2）高阶曲线分析

鉴于 $\mathrm{SO}_{2}$ 与 $\mathrm{NO}_{\mathrm{x}}$ 排放量与GDP的二次方程拟合相关系 数不高, 尝试高次幂进行相关性研究。通过不断尝试发现, 随着 $\mathrm{n}$ 值的不断增加, 拟合相关系数也不断提高, 当 $\mathrm{n}$ 为 6 的时候, $\mathrm{R}^{2}$ 分别达到了 0.9569 和 $0.9695, \mathrm{CO}_{2}$ 与 GDP的拟合 系数也随着 $\mathrm{n}$ 的增加而提高。可见，污染物与GDP之间的 关系均可以拟合为一元高次幂函数方程式, 且拟合程度均 较高, 充分体现了大气污染物与经济发展之间的关系。

表2 大气污染物排放总量与GDP的关系。

\begin{tabular}{lll}
\hline 污染物 & 模拟方程 & 相关系数 \\
\hline \multirow{3}{*}{$\mathrm{SO}_{2}$} & $\mathrm{y}=5 \mathrm{E}-15 \mathrm{x}^{3}-2 \mathrm{E}-08 \mathrm{x}^{2}+0.0102 \mathrm{x}+805.43$ & $\mathrm{R}^{2}=0.7945$ \\
& $\mathrm{y}=-2 \mathrm{E}-24 \mathrm{x}^{5}+3 \mathrm{E}-18 \mathrm{x}^{4}-2 \mathrm{E}-12 \mathrm{x}^{3}+6 \mathrm{E}-07 \mathrm{x}^{2}-0.0752 \mathrm{x}+5115$ & $\mathrm{R}^{2}=0.9438$ \\
& $\mathrm{y}=7 \mathrm{E}-30 \mathrm{x}^{6}-2 \mathrm{E}-23 \mathrm{x}^{5}+2 \mathrm{E}-17 \mathrm{x}^{4}-1 \mathrm{E}-11 \mathrm{x}^{3}+3 \mathrm{E}-06 \mathrm{x}^{2}-0.3479 \mathrm{x}+19187$ & $\mathrm{R}^{2}=0.9569$ \\
$\mathrm{NOx}$ & $\mathrm{y}=-1 \mathrm{E}-13 \mathrm{x}^{3}+1 \mathrm{E}-07 \mathrm{x}^{2}-0.051 \mathrm{x}+7359$ & $\mathrm{R}^{2}=0.8983$ \\
& $\mathrm{y}=4 \mathrm{E}-24 \mathrm{x}^{5}-8 \mathrm{E}-18 \mathrm{x}^{4}+6 \mathrm{E}-12 \mathrm{x}^{3}-3 \mathrm{E}-06 \mathrm{x}^{2}+0.5033 \mathrm{x}-36266$ & $\mathrm{R}^{2}=0.9286$ \\
& $\mathrm{y}=-8 \mathrm{E}-29 \mathrm{x}^{6}+2 \mathrm{E}-22 \mathrm{x}^{5}-2 \mathrm{E}-16 \mathrm{x}^{4}+1 \mathrm{E}-10 \mathrm{x}^{3}-5 \mathrm{E}-05 \mathrm{x}^{2}+8.481 \mathrm{x}-577005$ & $\mathrm{R}^{2}=0.9695$ \\
$\mathrm{CO}_{2}$ & $\mathrm{y}=4 \mathrm{E}-15 \mathrm{x}^{3}-7 \mathrm{E}-09 \mathrm{x}^{2}+0.0053 \mathrm{x}-311.2$ & $\mathrm{R}^{2}=0.9918$ \\
& $\mathrm{y}=-2 \mathrm{E}-26 \mathrm{x}^{5}+1 \mathrm{E}-20 \mathrm{x}^{4}+2 \mathrm{E}-14 \mathrm{x}^{3}-2 \mathrm{E}-08 \mathrm{x}^{2}+0.009 \mathrm{x}-631$ & $\mathrm{R}^{2}=0.9940$ \\
& $\mathrm{y}=3 \mathrm{E}-30 \mathrm{x}^{6}-6 \mathrm{E}-24 \mathrm{x}^{5}+6 \mathrm{E}-18 \mathrm{x}^{4}-3 \mathrm{E}-12 \mathrm{x}^{3}+7 \mathrm{E}-07 \mathrm{x}^{2}-0.0898 \mathrm{x}+4465.4$ & $\mathrm{R}^{2}=0.9982$ \\
\hline
\end{tabular}

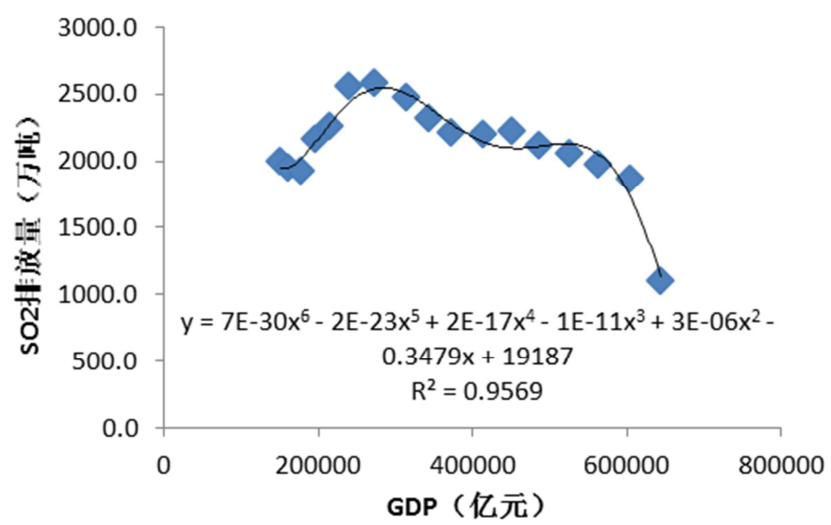

图6 $\mathrm{SO}_{2}$ 排放与GDP的高阶关系曲线。

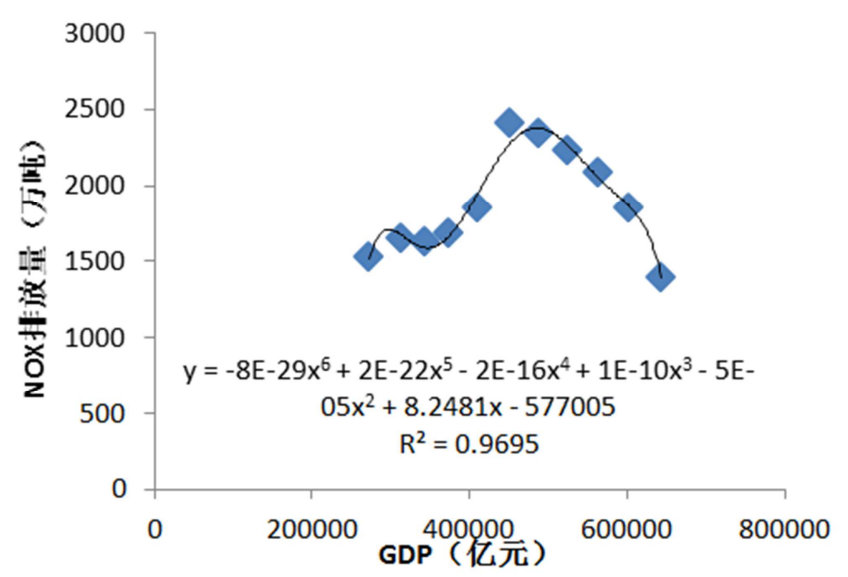

图7 $\mathrm{NO}_{\mathrm{x}}$ 排放与 GDP的高阶关系曲线。

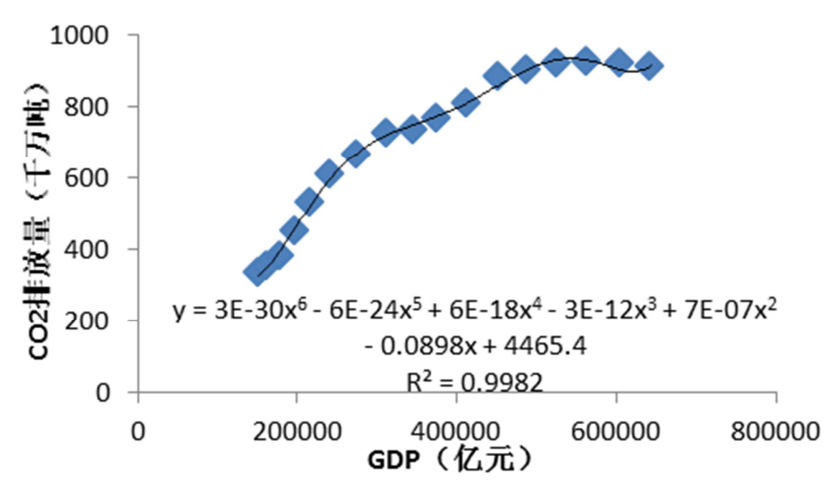

图 $8 \mathrm{CO}_{2}$ 排放与 $\mathrm{GDP}$ 的高阶关系曲线。

（3）各污染物曲线对比分析

由图3至图8可知, $\mathrm{SO}_{2}$ 和 $\mathrm{NO}_{\mathrm{x}}$ 与 $\mathrm{GDP}$ 的关系图较为相 似, 均已呈现倒U型, 随着经济的增长达到峰值后转而下 降, 但由于 $\mathrm{SO}_{2}$ 实施总量控制较早, 峰值出现也较早; $\mathrm{NOx}$ 实施总量控制后也随着经济的增长而排放量下降。 $\mathrm{CO}_{2}$ 与 $\mathrm{GDP}$ 关系与 $\mathrm{SO}_{2}$ 和 $\mathrm{NOx}$ 不同, 整体呈现正向增长关系, 即 随着GDP的增长而排放量增加, 但是在2015年起开始出现 下降态势。

\section{2. 脱钩弹性 $\mathrm{e}$ 的结果分析}

根据公式测算中国经济增长与 $\mathrm{SO}_{2} 、 \mathrm{NOx}$ 和 $\mathrm{CO}_{2}$ 排放 之间的脱钩弹性系数, 如表3所示。 
表3 Tapio弹性e计算结果汇总表。

\begin{tabular}{lllllll}
\hline 年份 & $\mathrm{E}_{\mathrm{S} 02, \mathrm{cDP}}$ & $\begin{array}{l}\text { 脱钩 } \\
\text { 状态 }\end{array}$ & $\mathrm{E}_{\mathrm{NOx}, \mathrm{GP}}$ & $\begin{array}{l}\text { 脱钩状 } \\
\text { 态 }\end{array}$ & $\mathrm{E}_{\mathrm{CO} 2, \mathrm{cDP}}$ & 脱钩状态 \\
\hline 2001 & -0.32 & 强 & - & - & 0.62 & 弱 \\
2002 & -0.13 & 强 & - & - & 0.95 & 弱 \\
2003 & 1.13 & 扩张 & - & - & 1.61 & 扩张 \\
2004 & 0.45 & 弱 & - & - & 1.58 & 扩张 \\
2005 & 1.18 & 扩张 & - & - & 1.27 & 扩张 \\
2006 & 0.13 & 弱 & - & - & 0.74 & 弱 \\
2007 & -0.38 & 强 & 0.57 & 弱 & 0.61 & 弱 \\
2008 & -0.69 & 强 & -0.13 & 强 & 0.21 & 弱 \\
2009 & -0.61 & 强 & 0.51 & 弱 & 0.55 & 弱 \\
2010 & -0.14 & 强 & 0.92 & 弱 & 0.56 & 弱 \\
2011 & 0.17 & 弱 & 2.64 & 扩张 & 0.90 & 弱 \\
2012 & -0.65 & 强 & -0.39 & 强 & 0.26 & 弱 \\
2013 & -0.50 & 强 & -0.69 & 强 & 0.36 & 弱 \\
2014 & -0.52 & 强 & -1.06 & 强 & 0.01 & 弱 \\
2015 & -0.96 & 强 & -1.90 & 强 & -0.10 & 强 \\
2016 & -10.92 & 强 & -5.22 & 强 & -0.07 & 强 \\
\hline
\end{tabular}

注: GDP以2010年不变价计, NOx自2006年开始全国范围统计。“弱”表 示弱脱钩, “强”表示强脱钩, “扩张”表示扩张性负脱钩。

总体来看, $\mathrm{SO}_{2}$ 和 $\mathrm{NOx}$ 排放与经济增长之间的脱钩关 系主要呈现强脱钩和弱脱钩关系, $\mathrm{CO}_{2}$ 和经济增长主要呈 现弱脱钩关系, 个别年份会呈现扩张性负脱钩。中国经济 一直呈现增长态势, 影响脱钩关系的因素主要取决于污染 物排放的变化量, 即污染物排放呈现增长态势还是下降态 势, 以及增长速率与经济增长速率的比较。其中, 污染物 排放与经济增长的脱钩程度随着宏观经济形势和政策调 控变化波动较大, 脱钩状态中, 出现的几次“拐点”, 均与 宏观经济形势和政策有关 $[25]$ 。如 $\mathrm{SO}_{2}$ 与经济增长的关系, 受2000年以后, 中国经济复苏的影响, 经济增长和工业化 速度加快, 工业经济进入高度发展阶段, 但整个经济依然 是以粗放型为特征, 产业结构不合理, 处于“三高一低”(高 投入、高能耗、高污染、低效率) 发展模式, 能源量增长 迅速, 导致污染物排放增速大于经济增速, 使脱钩弹性系 数持续增大, 2003 年超过 1 , 出现了扩张性负脱钩状态, 2004弹性系数也维持在较高状态, 2005 年再一次超过 1 , 直至2006年, 国务院发布了《关于加强节能工作的决定》, 2007年和2011年分别发布了《节能减排综合性工作方案》 和《“十二五”节能减排综合性工作方案》, 并制定了一系 列节能减排措施, 同时随着中国经济发展方式的转变、产 业结构的调整及发展环境友好经济, 国家重视节能减排, 出台了一系列相关措施, 使脱钩弹性系数下降, 维持在强 脱钩状态 $[26,27]$ 。

$\mathrm{NOx}$ 排放与经济增长的关系与 $\mathrm{SO}_{2}$ 类似, $\mathrm{NOx}$ 排放量 自2006年开始全面统计, 自2011年起机动车尾气污染物排 放情况与生活源分开单独统计, 且开始将其作为约束性指 标进行总量控制, 统计的完善和总量控制的实施, 导致 2011年NOx排放量统计数据增高, 出现了扩张性负脱钩状 态, 之后随着节能减排的实施和对NOx的总量控制, 使脱 钩弹性系数下降, 维持在强脱钩状态。

$\mathrm{CO}_{2}$ 排放与经济增长的关系, 落后于 $\mathrm{SO}_{2}$ 和 $\mathrm{NOx}$, 主 要受 $\mathrm{CO} 2$ 在在很长时期不涉及排放空间的稀缺性问题, 排 放规律更多的反映了经济发展的自然规律, 2006年之后, 随着国务院发布的《关于加强节能工作的决定》和《节能
减排综合性工作方案》等, 能源消耗的降低使 $\mathrm{CO} 2$ 排放增 速放缓 ${ }^{17]}$ 。且随着中国对气候变化的重视, 2014年制定了 第一部应对气候变化中长期规划《国家应对气候变化规划 （2014-2020年）》，2015年6月30日，正式公布中国国 家自主贡献预案: 《强化应对气候变化行动——国国家 自主贡献》, 2015 年 $\mathrm{CO}_{2}$ 排放总量首次出现下降, 与经济 呈现强脱钩关系。

\section{3. 两种模型对比分析}

在分析大气污染物排放与经济增长的关系时，环境 库兹涅茨曲线理论和Tapio脱钩理论都是最常用的理论, 二者在度量环境友好型经济时都有很好的适用性。本文 将EKC理论与脱钩理论同时用于中国大气污染物排放与 经济增长的实证分析, 在分别分析相关性及脱钩状态的 同时, 相互验证结果。对比结果显示, 两种方法得出的 中国大气污染物排放与经济增长的关系不冲突, 相互印 证吻合, 即 $\mathrm{EKC}$ 分析表明, 中国 $\mathrm{GDP}$ 与 $\mathrm{SO}_{2}$ 和 $\mathrm{NOx}$ 排放量 均相关, 且呈现倒 $\mathrm{U}$ 型关系, 大气污染物的排放量随着经 济的增长, 污染物排放逐步下降的态势, 这也表明中国 目前GDP与大气污染物排放出现脱钩现象; Tapio脱钩分 析显示, 中国“十一五”以来, 大气污染物排放与经济增 长整体呈现脱钩关系, 即随着经济的不断增长, $\mathrm{SO}_{2}$ 和 $\mathrm{NOx}$ 不断下降, 两种分析方法结论一致。

中国GDP与CO2排放量的关系, EKC分析表明, 中国 GDP与CO2排放量相关, 整体仍呈现正向线性关系, 但基 本处于拐点阶段; Tapio脱钩分析显示, 中国GDP与 $\mathrm{CO} 2$ 排放量的关系已由扩张性负脱钩状态经弱脱钩过渡为强 脱钩, 未来将进一步稳定在强脱钩状态, 两种分析方法结 论也一致。

中国GDP与 $\mathrm{SO}_{2} 、 \mathrm{NO}_{2}$ 的关系与 $\mathrm{CO}_{2}$ 的不同, 究其原因, 主要受国家政策导向影响, $\mathrm{SO}_{2} 、 \mathrm{NO}_{2}$ 作为主要污染物总 量控制指标实施多年, $\mathrm{SO}_{2} 、 \mathrm{NO}_{2}$ 已实现逐年下降, 而 $\mathrm{CO}_{2}$ 尚未作为污染物进行总量控制, 排放量直至2015年才出现 首次下降, 因此, 中国 $\mathrm{GDP}$ 和 $\mathrm{CO}_{2}$ 的关系曲线将落后于 $\mathrm{SO}_{2}$ 、 $\mathrm{NO}_{2}$, 但随着对 $\mathrm{CO}_{2}$ 控制力度的重视与加强, 中国 GDP与 $\mathrm{CO}_{2}$ 将逐步呈现倒U型关系, 实现强脱钩。

\section{5. 大气污染防治面临的新形势}

(1) 细颗粒物污染现象突出

2016年, 全国地级及以上城市 $\mathrm{SO}_{2}$ 和 $\mathrm{NO}_{2}$ 达标天数分 别达到 $99.5 \%$ 和 $98.4 \%$, 而 $\mathrm{PM}_{2.5}$ 达标天数为 $85.3 \%$, 以 $\mathrm{PM}_{2.5}$ 为首要污染物的天数占重度及以上污染天数的 $80.3 \%$ 。在 部分重污染天气, 甚至出现全国各城市细颗粒物均严重超 标的现象。为了应对细颗粒物污染严重等的问题, 中国已 开展大气污染源排放清单编制及大气污染源解析等工作, 以便在彻底排查摸底的基础上, 针对各区域的具体问题进 行对症下药。

（2）大气复合污染加剧

随着经济的发展, 大气污染物的种类不断增多, 各种 大气污染物在排放中及空气中, 形成大气复合污染, 即污 染物多种共存, 并相互结合或反应形成新的污染物质, 导 
致出现严重单位雾䨪天气, 甚至是光化学烟雾 ${ }^{[18]}$ 。近年来, 长三角、京津冀等地区雾霧现象频发。同时，由于各城市 机动车数量的增长较快, 使得 $\mathrm{NOx}$ 减排压力陡增, 而 $\mathrm{NOx}$ 作为 $\mathrm{PM}_{2.5}$ 和 $\mathrm{O}_{3}$ 的前体物, 也加剧了大气复合污染现象。

(3) 控制措施趋向联防联控

面对频繁出现的区域性污染，国家未来的大气污染防 控措施将逐步转向为区域性的联防联控措施, 各科研院所 等机构也将研究的目标投向联防联控技术等, 如对区域机 动车排放综合控制和绿色交通管理技术的研究, 对京津冀 等重点区域开展了大气污染联防联控技术集成与应用示 范, 将大气污染区域间的输送规律作为重点着力点, 并提 出要重点支撑区域重污染天气显著减少, $\mathrm{PM}_{2.5}$ 和臭氧等 污染物优先达标的目标。在未来的大气污染防治中，区域 性的大气污染控制管理机构或将逐步建立, 并发挥重要作 用。

\section{6. 应对建议}

\section{(1) 扩大推广 $\mathrm{SO}_{2}$ 和 NOx控制经验}

中国 $\mathrm{SO}_{2}$ 和 $\mathrm{NOx}$ 排放与 GDP已呈现倒U和脱钩状态, 污染防治的措施可逐步总结并应用于其他污染物控制中, 如对细颗粒物和 $\mathrm{CO}_{2}$ 的减排和总量控制等, 逐步在延续治 理 $\mathrm{SO}_{2}$ 和 $\mathrm{NOx}$ 的同时, 加强对颗粒物和 $\mathrm{CO}_{2}$ 的控制。

（2）加快推进大气污染协同控制

$\mathrm{SO}_{2} 、 \mathrm{NOx} 、 \mathrm{PM}^{2}$ 和 $\mathrm{CO}_{2}$ 很多时候出自同一个排污口, 在治理单个污染物的同时，更应加强多污染物的协同控 制, 积极研发和推广多污染物协同控制技术，合理选择 和搭配各治理措施, 使其不仅能起到治理重点控制的污 染物, 更能起到协同控制最优的效果, 进而减少污染物 排放, 特别死减少复合污染, 改善环境质量, 达到大气 污染与经济增长的进一步脱钩。

(3) 加快推进区域大气污染联防联控

加快推进区域大气污染联防联控研究与应用, 弄清污 染成因与传输影响机制, 建立区域大气污染预报预警机制, 建立区域联防联控机制与平台, 协商统筹, 解决因区域传 输造成的大气污染。

(4) 转变经济增长方式

要实现大气污染与经济增长的进一步脱钩, 改善环境 质量, 必须通过转变经济增长方式来实现。大力调整产业 结构, 以资源环境承载力以及节能减排目标为约束条件统 筹谋划未来产业发展方向, 推进经济结构的战略性调整和 升级。积极推进科技进步和创新, 增强自主创新能力, 鼓 励低碳环保技术创新, 推动创新成果转化推广。

\section{参考文献}

[1] Bruvoll A, Medin H. Factoring the environmental Kuznets curve Evidence from Norway [J]. Statistics Norway, Research Department in its series Discussion Papers, 2000(7), No.275.

[2] Bernard J T, Gavin M, Khalaf L, et al. The Environmental Kuznets Curve: Tipping Points, Uncertainty and Weak
Identification [J]. Cahiers De Recherché Create, 2011, 60(2):285 315.

[3] Ma S, Shi L. The Micro-foundations of the Environmental Kuznets Curve[J]. Fudan Journal of the Humanities \& Social Sciences, 2014, 7(3):471 482.

[4] DINDA S. Environmental Kuznets Curve hypothesis: a survey[J].Ecological economics, 2004, 49(4):431-455.

[5] Dogan E, Seker F. Determinants of $\mathrm{CO}_{2}$, Emissions in the European Union: The Role of Renewable and Non renewable Energy[J]. Renewable Energy, 2016, 94: 429439.

[6] Menendez A J L, Prezr R, Moreno B. Environmental Costs and Renewable Energy:Re - visiting the Environmental Kuznets Curve [J]. Journal of Environmental Management, 2014, 145(1):368-373.

[7] Hauff M V, Mistri A. Environmental Kuznets Curve (EKC): Implications on Economic Growth, Access to Safe Drinking Water and Ground Water Utilization in India[J]. Journal of Fish Biology, 2015,79(6):1563 1591.

[8] De Bruyn S M. Economic growth and the environment[J]. Dordrecht: Kluwer Academic Publishers, 2000, 62.

[9] Vehmas J, Kaivo-oja J, Luukkanen J. Global trends of linking environmental stress and economic growth[J]. TUTU publications, 2003, 7: 1 25.

[10] Tapio P. Towards a Theory of Decoupling: Degrees of Decoupling in the EU and the Case of Road Traffic in Finland between 1970 and 2001 [J]. Journal of Transport Policy, 2005, (12): 137 151.

[11] GRAY D, ANABLE J, ILLINGWORTH L, et al. Decoupling the link between economic growth,transport growth and carbon emissions in Scotland [R]. Edinburgh: The Scottish Executive, 2006.

[12] 王敏, 黄滢.中国的环境污染与经济增长[J].经济学, 2015, 14(2): 557 578。

[13] 范丹.中国二氧化碳EKC曲线扩展模型的空间计量分析 [J]. 宏观经济研究, 2014(5):83 91。

[14] 罗波, 张金锁，张伟等.资源富集区经济发展与环境污染的 $\mathrm{EKC}$ 实证研究一以扇贝地区为例 [J]. 中国煤炭, 2017,43(5):10 15。

[15] 尚杰, 李新, 邓雁云.基于EKC的农业经济增长与农业面源 污染的关系分析一以黑龙江省为例 [J].生态经济, 2017, 33(6):157 166。

[16] 王光升, 郭佩芳, 谭映宇, 等. 基于单位根检验的沿海地区 经济增长与海洋环境污染面板数据 $\mathrm{EKC}$ 分析 [J].海洋环境 科学, 2014, 03: 425 430。

[17] 李斌, 曹万林.经济发展与环境污染的脱钩分析 [J].经济学动 态, 2014, (7):48 56。

[18] 彭佳雯, 黄贤金, 钟太洋, 等.中国经济增长与能源碳排放 的脱钩研究 [J].资源科学，2011，33(04)：626 633。 
[19] 方佳敏, 林基.中国工业行业经济增长与二氧化碳排放的脱 钩效应一基于工业行业数据的经验证据 [J].科技管理研究, 2015，20：243 248。

[20] 夏勇, 胡雅蓓. 经济增长与环境污染脱钩的因果链分解及内 外部成因研究一来自中古 30 个省份的工业 $\mathrm{SO}_{2}$ 排放数据 $[\mathrm{J}]$. 产业经济研究, 2017, (5): 100 113。

[21] 夏勇, 钟茂初. 经济发展与环境污染脱钩理论及EKC假说的 关系一兼论中国地级城市的脱钩划分 [J].中国人口.资源与 环境, 2016, 26(10): 8 16。

[22] Kuznets S. Economic growth and income inequality [J]. American Economic Review, 1955, (45): 1-28.
[23] Grossman G. and Krueger A. Environmental impacts of the North American Free Trade Agreement [D]. NBER, working paper, No.3914, 1991.

[24] OECD. Indicators to Measure Decoupling of Environmental Pressures From Economic Growth [R]. Paris: OECD, 2002.

[25] 杨占红, 吕连宏, 曹宝等. 国际能源消费特征比较分析及中 国发展建议[J].地球科学进展, 2016, 31(1): 94 102。

[26] 杨占红, 裴荣荣, 罗宏等. 国际碳排放特征演进及中国应对 建议 [J].气候变化研究进展, 2016, 12(03): 185 192。

[27] 谢振明.大气污染治理形势及其存在问题和建议 [J]. 能源与 环境, 2016, (1): 53 54。 